\title{
Photon directional profile from stimulated decay of axion clouds with nonspherical axion spatial distributions
}

\author{
Liang Chen $\oplus^{*}$ and Thomas W. Kephart $\oplus^{\dagger}$ \\ Department of Physics and Astronomy, Vanderbilt University, \\ Nashville, Tennessee 37235, USA
}

(Received 8 May 2020; accepted 5 October 2020; published 11 November 2020)

\begin{abstract}
We model clusters of axions with spherically symmetric momentum but arbitrary spatial distributions and study the directional profile of photos produced in their evolution through spontaneous and stimulated decay of axions via the process $a \rightarrow \gamma+\gamma$. Several specific examples are presented.
\end{abstract}

DOI: 10.1103/PhysRevD.102.096010

\section{INTRODUCTION}

Axions are copiously produced at the QCD phase transition. A possible way to detect these cosmological axions is through the observation of lasing axion clouds (clumps). If axions are a component of the cold dark matter (CDM), then they can form density perturbations in the early Universe. If the overdense regions have high enough number density, then ambient photons from the cosmic microwave background (CMB), or from spontaneous axion decays, can induce stimulated axion decay within the clumps; i.e., the axions can lase [1-3].

Besides the initial clumps, other axion structures can form. The initial density perturbations can infall and evolve to form caustics [4], which have complicated geometries. Yet another possibility is that axions can be produced after the formation of primordial black holes (PBHs). Such black holes can be the results of various early Universe processes, from cosmic string or domain wall singularities to density perturbations. However they are formed, if they have sufficient angular momentum, either initially or from mergers, then superradiance can occur causing axions to populate an $n, l, m=2,1,1$ hydrogenlike orbit around them if the axion Compton wavelength is comparable to the PBHs' radius. If the axion density is high enough they can lase [5]. The process can saturate, stop and then repeat in a way similar to what has been seen for fast radio bursts.

Lasing in the PBH superradiance case has so far only been approximated using the spherically symmetric model [5]. In this work and in [6] we point the way to an

\footnotetext{
*bqipd@pm.me

tom.kephart@gmail.com
}

Published by the American Physical Society under the terms of the Creative Commons Attribution 4.0 International license. Further distribution of this work must maintain attribution to the author(s) and the published article's title, journal citation, and DOI. Funded by SCOAP ${ }^{3}$. improving this approximation using multipole expansions of the spatial and momentum space distributions to more closely represent the physical axion distributions expected around a PBH.

There are other works that investigate nonspherically symmetric distributions of axion clumps based on field theory. QCD axion clumps with conventional couplings can undergo resonant decay for sufficiently large angular momentum [7]. Radio emission from QCD axions occurs in the context of Bose stars if axion-photon coupling is large [8]. Reference [9] gives numerical description of laserlike emission from axion clouds. Detailed studies of axionic instability induced by electromagnetic field deformations of the Kerr-Newman geometry by axions, and periodic bursts of light from superradiant growth are carried out in [10]. Reviews of basic axion physics, axion cosmology, axion clusters and the most recent axion search results can be found in [11-19].

\section{PHOTON ANGULAR DISTRIBUTION}

In $[1,3]$ nonrelativistic axions of mass $m_{a}$ were contained in a ball of radius $R$, with a maximum momentum value of $p_{\max } \approx m_{a} \beta$. Here we allow a nonspherically symmetric spatial distribution $X(\theta, \phi)$ to modify the axion clouds model previously studied, with the aim of finding the angular distribution $Y(\theta, \phi)$ of photons resulted from decays of axions, providing that there is some outside constraint (e.g., a gravitational field or self-interactions) that can keep the axions in the initial spatial distribution. (As we will see, this endeavor will fail, but understanding the failure will teach us how to solve the problem.) For such an axion distribution, assuming it factorizes, the occupation number $f_{a}(p, r, \theta, t)$ and number densities $n_{a}(r, \theta, t)$ can be written

$$
f_{a}(p, r, \theta, t)=f_{a c}(t) \Theta\left(p_{\max }-p\right) \Theta(R-r) X(\theta),
$$


and

$$
\begin{aligned}
n_{a}(r, \theta, t) & =\int \frac{d^{3} p}{(2 \pi)^{3}} f_{a}(p, r, \theta, t), \\
& =\frac{m_{a}^{3} \beta^{3}}{6 \pi^{2}} f_{a c}(t) \Theta(R-r) X(\theta), \\
& =n_{a c}(t) \Theta(R-r) X(\theta),
\end{aligned}
$$

where we can translate between the two with

$$
f_{a c}(t)=\frac{6 \pi^{2}}{m_{a}^{3} \beta^{3}} n_{a c}(t)
$$

Here and elsewhere we use the short-hand notation $X(\theta)$ for $X(\theta, \phi)$, likewise for $Y, f$, and $n$.

The photons are contained in a ball of radius $R$, a momentum spherical shell of inner and outer radius $k_{-}=\frac{m_{a} \gamma}{2}(1-\beta)$ and $k_{+}=\frac{m_{a} \gamma}{2}(1+\beta)$, respectively [3], where we use $\beta=v / c$.

$$
f_{\lambda}(k, r, \theta, t)=f_{\lambda c}(t) \Theta\left(k_{+}-k\right) \Theta\left(k-k_{-}\right) \Theta(R-r) Y(\theta),
$$

and

$$
\begin{aligned}
n_{\lambda}(r, \theta, t) & =\int \frac{d^{3} k}{(2 \pi)^{3}} f_{\lambda}(k, r, \theta, t), \\
& =f_{\lambda c}(t) \Theta(R-r) Y(\theta) \frac{V_{k}}{8 \pi^{3}}, \\
& =n_{\lambda c}(t) \Theta(R-r) Y(\theta),
\end{aligned}
$$

with

$$
f_{\lambda c}(t)=\frac{8 \pi^{2}}{m_{a}^{3} \beta} n_{\lambda c}(t)
$$

where $f_{\lambda}(k, r, \theta, t)$ and $n_{\lambda}(r, \theta, t)$ are the photon occupation number and photon number density, of helicity $\lambda= \pm 1$ respectively, which are related by Eq. (6), and $V_{k}$ is the volume of the momentum spherical shell

$V_{k}=\int_{k_{-}}^{k_{+}} d^{3} k=4 \pi\left(\frac{m_{a} \gamma}{2}\right)^{2}\left(k_{+}-k_{-}\right)=\pi m_{a}^{3} \beta \gamma^{3} \approx \pi m_{a}^{3} \beta$.

We assume that the number density of each helicity state is the same, so the total photon number density $n_{\gamma}$ can be written as

$$
\begin{aligned}
n_{\gamma}(r, \theta, t) & =n_{\gamma c}(t) \Theta(R-r) Y(\theta)=n_{+}(r, \theta, t)+n_{-}(r, \theta, t) \\
& =\left[n_{+c}(t)+n_{-c}(t)\right] \Theta(R-r) Y(\theta), \\
n_{+c}(t) & =n_{-c}(t), n_{\gamma c}(t)=2 n_{\lambda c}(t)
\end{aligned}
$$

which defines $n_{x c}$. Hence the coefficient of the total photon number density is just two times that of photon number density of each helicity state.

The evolution relation between axion and photon occupation numbers is [see Eqs. (13) of [3]]

$$
\begin{aligned}
\frac{d f_{\lambda}(k)}{d t}= & \frac{m_{a} \Gamma_{a}}{k^{2}} \int_{\frac{m_{a}^{2}}{4 k}} d k_{1} \times\left\{f_{a}\left(k+k_{1}\right)\left[1+f_{\lambda}(k)+f_{\lambda}\left(k_{1}\right)\right]\right. \\
& \left.-f_{\lambda}(k) f_{\lambda}\left(k_{1}\right)\right\}
\end{aligned}
$$

where $f_{\lambda}(k)$ and $f_{\lambda}\left(k_{1}\right)$ are photon occupation numbers of momentum $k$ and $k_{1}$, respectively, $\Gamma_{a}$ is the spontaneous axion decay rate and $\tau_{a}=1 / \Gamma_{a}$ is the decay timescale. Other variables in $f_{\lambda}(k)$ and $f_{\lambda}\left(k_{1}\right)$; i.e., $r, \theta$, $t$, are the same since they share the same spacetime. $f_{a}\left(k+k_{1}\right)$ is the axion occupation number of momentum $k+k_{1}$.

This evolution equation can be integrated over $k$ and $k_{1}$ phase space to yield (see the Appendix)

$$
\begin{aligned}
\frac{d n_{\lambda}}{d t}= & \Theta(R-r) \frac{m_{a}^{3} \Gamma_{a}}{8 \pi^{2}}\left\{\left[f_{a c}\left(X+2 f_{\lambda c} X Y\right)-f_{\lambda c}^{2} Y^{2}\right]\right. \\
& \left.\times\left[2 \gamma^{2} \beta-\ln \left(\frac{1+\beta}{1-\beta}\right)\right]-f_{\lambda c}^{2} Y^{2} \times\left(2 \gamma^{2} \beta^{2}\right)\right\} .
\end{aligned}
$$

Now we employ the nonrelativistic approximation $(\beta \ll 1)$

$$
\begin{aligned}
2 \gamma^{2} \beta & =\frac{2 \beta}{1-\beta^{2}} \approx 2 \beta\left(1+\beta^{2}\right)=2 \beta+2 \beta^{3}, \\
\ln \left(\frac{1+\beta}{1-\beta}\right) & \approx 2 \beta+\frac{2 \beta^{3}}{3},
\end{aligned}
$$$$
2 \gamma^{2} \beta^{2} \approx 2 \beta^{2},
$$

to arrive at

$$
\begin{aligned}
\frac{d n_{\lambda}}{d t}= & \Theta(R-r) \times \frac{m_{a}^{3} \Gamma_{a} \beta^{2}}{6 \pi^{2}}\left\{f_{a c}\left(X+2 f_{\lambda c} X Y\right) \beta\right. \\
& \left.-\left(\beta+\frac{3}{2}\right) f_{\lambda c}^{2} Y^{2}\right\} .
\end{aligned}
$$

Substituting the derived relations (3) and (5) into (9) yields

$$
\begin{aligned}
\frac{d n_{\lambda}}{d t}= & \Gamma_{a} \Theta(R-r) \times\left[n_{a c}\left(X+\frac{16 \pi^{2} n_{\lambda c}}{\beta m_{a}^{3}} X Y\right)\right. \\
& \left.-\frac{32 \pi^{2} n_{\lambda c}^{2}}{3 m_{a}^{3}}\left(\beta+\frac{3}{2}\right) Y^{2}\right] .
\end{aligned}
$$


Taking into consideration photon surface losses [3]

$$
\left(\frac{d n_{\lambda}}{d t}\right)_{\text {surface loss }}=-\frac{3 c n_{\lambda}}{2 R}=-\frac{3 c}{2 R} n_{\lambda c} \Theta(R-r) Y(\theta),
$$

we have an equation that gives the number density for each helicity state

$$
\begin{aligned}
\frac{d n_{\lambda}}{d t}= & \Theta(R-r) \times\left[\frac{n_{a c}}{\tau_{a}} X(\theta)+\frac{16 \pi^{2} n_{a c} n_{\lambda c}}{\beta m_{a}^{3} \tau_{a}} X(\theta) Y(\theta)\right. \\
& \left.-\frac{32 \pi^{2} n_{\lambda c}^{2}}{3 m_{a}^{3} \tau_{a}}\left(\beta+\frac{3}{2}\right) Y(\theta)^{2}-\frac{3 c n_{\lambda c}}{2 R} Y(\theta)\right],
\end{aligned}
$$

where we are assuming, as was shown in (7), that the total number density of photons is twice that of the individual helicity states. Therefore the rate of change of total number density of photons is

$$
\begin{aligned}
\frac{d n_{\gamma}}{d t}= & \Theta(R-r) \times\left[2 \frac{n_{a c}}{\tau_{a}} X(\theta)+\frac{16 \pi^{2} n_{a c} n_{\gamma c}}{\beta m_{a}^{3} \tau_{a}} X(\theta) Y(\theta)\right. \\
& \left.-\frac{16 \pi^{2} n_{\gamma c}^{2}}{3 m_{a}^{3} \tau_{a}}\left(\beta+\frac{3}{2}\right) Y^{2}(\theta)-\frac{3 c n_{\gamma c}}{2 R} Y(\theta)\right]
\end{aligned}
$$

Since from (7)

$$
\frac{d n_{\gamma}}{d t}=\frac{d n_{\gamma c}}{d t} \Theta(R-r) Y(\theta),
$$

if we drop the step function $\Theta(R-r)$ we have an equation for the coefficient of total number density of photon

$$
\begin{aligned}
\frac{d n_{\gamma c}}{d t}= & 2 \frac{n_{a c}}{\tau_{a}} \frac{X(\theta)}{Y(\theta)}+\frac{16 \pi^{2} n_{a c} n_{\gamma c}}{\beta m_{a}^{3} \tau_{a}} X(\theta) \\
& -\frac{16 \pi^{2} n_{\gamma c}^{2}}{3 m_{a}^{3} \tau_{a}}\left(\beta+\frac{3}{2}\right) Y(\theta)-\frac{3 c n_{\gamma c}}{2 R} .
\end{aligned}
$$

From the first to the last term on the right-hand side (rhs) of the equation, the terms account for spontaneous decay of axions, photon stimulated decay of axions, backreaction of photons, and surface loss of photons, respectively. Following a similar approach, we obtain an equation regarding the coefficient of total number density of axions

$$
\frac{d n_{a c}}{d t}=-\frac{n_{a c}}{\tau_{a}} \frac{X(\theta)}{Y(\theta)}-\frac{8 \pi^{2} n_{a c} n_{\gamma c}}{\beta m_{a}^{3} \tau_{a}} X(\theta)+\frac{8 \pi^{2} n_{\gamma c}^{2} \beta}{3 m_{a}^{3} \tau_{a}} Y(\theta) .
$$

The third term on the rhs of (14) is proportional to $\beta$, while the third term on the rhs of (13) has a factor of $\left(\beta+\frac{3}{2}\right)$. Keeping track of two parts of axions generated from the back reacting photons, we find that the $\frac{3}{2}$ in the third term on the rhs of (13) represents sterile axions [3] and it should have been and was excluded in the derivation of (14).

The left-hand sides (lhs) of (13) and (14) have no $\theta$ dependence, but the rhs does. $X(\theta)=Y(\theta)$ will not make (13) and (14) valid simultaneously. So even if there is some outside constraint which can keep the axions fixed in the $X(\theta)$ distribution, the photons cannot have the same distribution, i.e., $Y(\theta) \neq X(\theta)$.

There is no simple way to find a closed form for $Y(\theta)$ because the lhs of Eqs. (13) and (14) have no $\theta$ dependence, while the $\theta$ dependences on the rhs of these equations are different. This suggests the possibility that $Y(\theta)$ may be found as a series expansion in $X(\theta)$. As a first test of this idea we replaced the general form $X(\theta)$ with $\sin \theta$ to study the distribution with more axions accumulated near the equatorial plane with few near the polar area, aiming at matching orders of $\sin \theta$ on each side of equations. But this fails as it turns out that $\sin ^{n} \theta(n \in \mathbb{Z})$ is not an orthogonal set of functions and thus the calculation leads to contradictions. Therefore, we must expand the occupation numbers and number density in terms of a full set of orthogonal functions. We do this in the next section where we choose the set to be the real spherical harmonics.

\section{REAL SPHERICAL HARMONICS EXPANSION}

The setup here is similar to the previous discussion except that the axion and photon occupation numbers and number densities have coefficients labeled by order indices $l$ and $m$. Occupation number is treated as homogeneous in the radial direction, see [9] for a nonhomogeneous radial distribution that describes superradiance. We plan to apply the results obtained here to improve the approximations used in [5]. Our formalism does not fully incorporate the physical mechanism of superradiance or gravity. (It is more a decay model for scalar particles that is built on statistics than a superradiance-blast simulation model.) For the axions

$$
\begin{aligned}
f_{a}(p, r, \Omega, t) & =\sum_{l m} f_{a l m}(t) Y_{l m}(\Omega) \Theta\left(p_{\max }-p\right) \Theta(R-r), \\
n_{a}(r, \Omega, t) & =\sum_{l m} n_{a l m}(t) Y_{l m}(\Omega) \Theta(R-r), \\
f_{\text {alm }}(t) & =\frac{6 \pi^{2}}{m_{a}^{3} \beta^{3}} n_{\text {alm }}(t),
\end{aligned}
$$

where we have set

$$
f_{a c}(t) X(\theta)=\sum_{l m} f_{a l m}(t) Y_{l m}(\Omega) .
$$

Note that if $n_{a}$ describes a real physical number density, it has to be real and positive and be able to be put into the form

$$
n_{a}=\Theta(R-r)\left(\sum_{l^{\prime} m^{\prime}} n_{a l^{\prime}}^{m^{\prime}} Y_{l^{\prime}}^{m^{\prime}}\right)^{*}\left(\sum_{l m} n_{a l}^{m} Y_{l}^{m}\right),
$$


using Clebsch-Gordan coefficients, where $Y_{l}^{m}$ are complex spherical harmonics. This also applies to photons. For instance, $\quad n_{a}=\Theta(R-r) n_{a 20} Y_{20}(\Omega)$ cannot describe a physical number density since it cannot be written as a product of complex spherical harmonics and its conjugate. But

$$
n_{a}=\Theta(R-r) b(t) Y_{00}-\frac{1}{\sqrt{5}} b(t) Y_{20}
$$

does describe a physical number density since we can write it as a product of complex spherical harmonics and its conjugate, $n_{a} \sim c(t) Y_{1}^{1 *} Y_{1}^{1}$. The photon occupation number and number density, and the relation between them are

$$
\begin{aligned}
f_{\lambda}(k, r, \Omega, t)= & \sum_{l m} f_{\lambda l m}(t) Y_{l m}(\Omega) \\
& \times \Theta(R-r) \Theta\left(k_{+}-k\right) \Theta\left(k-k_{-}\right), \\
n_{\lambda}(r, \Omega, t)= & \sum_{l m} n_{\lambda l m}(t) Y_{l m}(\Omega) \Theta(R-r), \\
f_{\lambda l m}(t)= & \frac{8 \pi^{2}}{m_{a}^{3} \beta} n_{\lambda l m}(t) .
\end{aligned}
$$

We also write

$$
\begin{aligned}
n_{\gamma}(r, \Omega, t) & =\sum_{l m}\left[n_{+l m}(t)+n_{-l m}(t)\right] Y_{l m}(\Omega) \Theta(R-r), \\
& =\sum_{l m} n_{\gamma l m}(t) Y_{l m}(\Omega) \Theta(R-r), \\
n_{+l m}(t) & =n_{-l m}(t), \quad n_{\gamma l m}(t)=2 n_{\lambda l m}(t),
\end{aligned}
$$

where similar the axion case we have set

$$
f_{\lambda c}(t) Y(\theta)=\sum_{l m} f_{\lambda l m}(t) Y_{l m}(\Omega) .
$$

Following the steps from the previous general discussion, we have an equation similar to (13) for each choice of $l m$

$$
\begin{aligned}
\frac{d n_{\gamma l m}(t)}{d t}= & 2 \frac{n_{a l m}}{\tau_{a}}+\frac{16 \pi^{2}}{\beta m_{a}^{3} \tau_{a}} E_{l m} \\
& -\frac{16 \pi^{2}}{3 m_{a}^{3} \tau_{a}}\left(\beta+\frac{3}{2}\right) F_{l m}-\frac{3 c}{2 R} n_{\gamma l m}(t),
\end{aligned}
$$

where $E_{l m}$ and $F_{l m}$ are defined through

$$
\begin{aligned}
n_{a}(\Omega, t) n_{\gamma}(\Omega, t) & =\sum_{l^{\prime} m^{\prime} l^{\prime \prime} m^{\prime \prime}} n_{a l^{\prime} m^{\prime}} n_{\gamma l^{\prime \prime} m^{\prime \prime}} Y_{l^{\prime} m^{\prime}} Y_{l^{\prime \prime} m^{\prime \prime}}, \\
& =\sum_{l m} E_{l m} Y_{l m},
\end{aligned}
$$

and

$$
\begin{aligned}
{\left[n_{\gamma}(\Omega, t)\right]^{2} } & =\sum_{l^{\prime} m^{\prime} l^{\prime \prime} m^{\prime \prime}} n_{\gamma l^{\prime} m^{\prime}} n_{\gamma l^{\prime \prime} m^{\prime \prime}} Y_{l^{\prime} m^{\prime}} Y_{l^{\prime \prime} m^{\prime \prime}}, \\
& =\sum_{l m} F_{l m} Y_{l m} .
\end{aligned}
$$

We also have equations similar to Eq. (14) for each choice of $l m$ with regard to the changing number density of axions. The equation includes components representing spontaneous decay, stimulated decay, and backreaction with sterile axions excluded

$$
\frac{d n_{a l m}(t)}{d t}=-\frac{n_{a l m}}{\tau_{a}}-\frac{8 \pi^{2}}{\beta m_{a}^{3} \tau_{a}} E_{l m}+\frac{8 \pi^{2} \beta}{3 m_{a}^{3} \tau_{a}} F_{l m} .
$$

The sterile axions evolve according to

$$
\frac{d n_{s l m}(t)}{d t}=\frac{4 \pi^{2}}{m_{a}^{3} \tau_{a}} F_{l m}
$$

The rate of change of photon number density component can be expressed in terms of the changing components of normal axion and sterile axion, and the components of surface loss

$$
\frac{d n_{\gamma l m}(t)}{d t}=-2\left[\frac{d n_{a l m}(t)}{d t}+\frac{d n_{s l m}(t)}{d t}\right]-\frac{3 c}{2 R} n_{\gamma l m}(t) .
$$

We now proceed to explore some example choices of initial axion distributions.

\section{EXAMPLES}

\section{A. $Y_{00}$ distribution}

As a first example we consider the spherical symmetric axion distribution where the only nonzero component of axion number density is $n_{a 00}$,

$$
n_{a}=\Theta(R-r) n_{a 00} Y_{00}(\Omega)
$$

then

$$
n_{a l m}=0 \quad(\operatorname{lm} \neq 00) .
$$

This simplifies Eq. (17) to

$$
n_{a}(\Omega, t) n_{\gamma}(\Omega, t)=\sum_{l m} n_{a 00} Y_{00} n_{\gamma l m} Y_{l m} .
$$

In addition, there is now a relationship between $E_{l m}$ and $n_{\gamma l m}$,

$$
E_{l m}=n_{a 00} Y_{00} n_{\gamma l m}
$$

Equation (19) is also simplified for $l m \neq 00$ to 


$$
0=0-\frac{8 \pi^{2}}{\beta m_{a}^{3} \tau_{a}} E_{l m}+\frac{8 \pi^{2} \beta}{3 m_{a}^{3} \tau_{a}} F_{l m}
$$

which reduces to

$$
F_{l m}=\frac{3}{\beta^{2}} E_{l m} \quad(l m \neq 00) .
$$

Substitute (22) and (23) into Eq. (18) gives, upon splitting of 00 pieces, the two forms of (18)

$$
\begin{aligned}
{\left[n_{\gamma}(\Omega, t)\right]^{2} } & =F_{00} Y_{00}+\frac{3}{\beta^{2}} n_{a 00} Y_{00} \sum_{l m \neq 00} n_{\gamma l m} Y_{l m}, \\
F_{l m} & =\frac{3}{\beta^{2}} E_{l m} \quad(l m \neq 00),
\end{aligned}
$$

and

$$
\begin{aligned}
{\left[n_{\gamma}(\Omega, t)\right]^{2}=} & \frac{n_{\gamma 00} n_{\gamma 00}}{2 \sqrt{\pi}} Y_{00}+2 n_{\gamma 00} Y_{00} \sum_{l m \neq 00} n_{\gamma l m} Y_{l m} \\
& +\sum_{l^{\prime} m^{\prime} l^{\prime \prime} m^{\prime \prime} \neq 0000} n_{\gamma l^{\prime} m^{\prime}} n_{\gamma l^{\prime \prime} m^{\prime \prime}} Y_{l^{\prime} m^{\prime}} Y_{l^{\prime \prime} m^{\prime \prime}} .
\end{aligned}
$$

The most conspicuous solution to the equation is

$$
F_{00}=\frac{n_{\gamma 00} n_{\gamma 00}}{2 \sqrt{\pi}}, \quad n_{\gamma l m}=0 \quad(\operatorname{lm} \neq 00),
$$

where the only nonzero component of photon number density is $n_{\gamma 00}$. So if there is spherical symmetry in the axion distribution, then spherical symmetry also exist in photon distribution.

Now we argue that this is the only solution of the finite spherical harmonics series. Suppose that the highest spherical harmonics in the photon number density $n_{\gamma}(\Omega, t)$ is $Y_{l_{a} m_{a}}$. According to (24) and taking the $Y_{00}$ as a number, the highest spherical harmonics in $\left[n_{\gamma}(\Omega, t)\right]^{2}$ is also $Y_{l_{a} m_{a}}$. However, according to (25), the highest spherical harmonics in $\left[n_{\gamma}(\Omega, t)\right]^{2}$ is going to be $Y_{2 l_{a} 2 m_{a}}$. This contradiction can only be resolved when $n_{\gamma l m}=0(\operatorname{lm} \neq 00)$; i.e., the photon number density retains spherical symmetry.

The reason why this is the only finite series case is that the $Y_{00}$ distribution of axions mathematically requires the photons to couple in a specific way that retains the $Y_{00}$ distribution of axions, as is implied by Eqs. (22) and (23).

Now we know all the coupling coefficients $E_{l m}$ and $F_{l m}$,

$$
E_{l m}=\frac{n_{a 00} n_{\gamma 00}}{2 \sqrt{\pi}} \delta_{l 0} \delta_{m 0}, \quad F_{l m}=\frac{n_{\gamma 00} n_{\gamma 00}}{2 \sqrt{\pi}} \delta_{l 0} \delta_{m 0} .
$$

Equations (16), (19), and (20) reduce to the Eqs. (34'), (37'), and (38') in [3] given that

$$
n_{\gamma 00}=2 \sqrt{\pi} n_{\gamma}, \quad n_{a 00}=2 \sqrt{\pi} n_{a},
$$

because it is the $n_{\gamma 00} Y_{00}$ that describes the photon number density. Hence we have checked the spherically symmetric model results given in [3].

As pointed out above, this next example will not be physical, but included as it is informative and useful in what follows.

\section{B. $Y_{20}$ distribution}

For a $Y_{20}$ axion distribution the only nonzero component of the axion number density is $n_{a 20}$,

$$
n_{a}=\Theta(R-r) n_{a 20} Y_{20}(\Omega),
$$

so that

$$
n_{\text {alm }}=0 \quad(\operatorname{lm} \neq 20) .
$$

Equation (19) is simplified for $\operatorname{lm} \neq 20$, to

$$
0=0-\frac{8 \pi^{2}}{\beta m_{a}^{3} \tau_{a}} E_{l m}+\frac{8 \pi^{2} \beta}{3 m_{a}^{3} \tau_{a}} F_{l m},
$$

which reduces to

$$
F_{l m}=\frac{3}{\beta^{2}} E_{l m} \quad(l m \neq 20) .
$$

The nonzero component $n_{a 20}$ of axion number density evolves via

$$
\frac{d n_{a 20}(t)}{d t}=-\frac{n_{a 20}}{\tau_{a}}-\frac{8 \pi^{2}}{\beta m_{a}^{3} \tau_{a}} E_{20}+\frac{8 \pi^{2} \beta}{3 m_{a}^{3} \tau_{a}} F_{20} .
$$

The photon number density component $n_{\gamma 20}$ growth rate is

$$
\begin{aligned}
\frac{d n_{\gamma 20}(t)}{d t}= & 2 \frac{n_{a 20}}{\tau_{a}}+\frac{16 \pi^{2}}{\beta m_{a}^{3} \tau_{a}} E_{20}-\frac{16 \pi^{2}}{3 m_{a}^{3} \tau_{a}}\left(\beta+\frac{3}{2}\right) F_{20} \\
& -\frac{3 c}{2 R} n_{\gamma 20}(t),
\end{aligned}
$$

while the other photon number density component $n_{\gamma l m}(l m \neq 20)$ evolve as

$$
\frac{d n_{\gamma l m}(t)}{d t}=-\frac{8 \pi^{2}}{m_{a}^{3} \tau_{a}} F_{l m}-\frac{3 c}{2 R} n_{\gamma l m}(t)
$$

Since no spontaneous decay from axion feeds into these components, they are negligible. This example is not physical because a number density of the form $Y_{20}$ becomes negative in some regions. It is included here for demonstration purpose. Even though this example is not physical, it is needed because it is a component of the next example which is physical and motivated by superradiance. 


\section{C. $Y_{1}^{ \pm 1 *} Y_{1}^{ \pm 1} \sim \sin ^{2} \theta$ distribution}

A $\sin ^{2} \theta$ distribution is torodial and is positive definite everywhere, and hence can represent a physical distribution of particles. For this case the only nonzero components of the axion number density are $n_{a 00}$ and $n_{a 20}$, so we can write $n_{a}(r, \theta, t)$ in several useful forms

$$
\begin{aligned}
n_{a} & =\Theta(R-r) n_{a}(t) \sin ^{2} \theta, \\
& =\Theta(R-r) n_{a}(t) \frac{4 \sqrt{\pi}}{3}\left(Y_{00}-\frac{1}{\sqrt{5}} Y_{20}\right), \\
& =\Theta(R-r)\left[n_{a 00}(t) Y_{00}+n_{a 20}(t) Y_{20}\right] .
\end{aligned}
$$

The relation between $n_{a 00}$ and $n_{a 20}$ is

$$
n_{a 20}(t)=-\frac{n_{a 00}(t)}{\sqrt{5}} .
$$

Similar to previous examples, we find that for components other than 00 and 20

$$
F_{l m}=\frac{3}{\beta^{2}} E_{l m} \quad(l m \neq 00,20),
$$

so that the components of photon number density evolve as

$$
\frac{d n_{\gamma l m}(t)}{d t}=-\frac{8 \pi^{2}}{m_{a}^{3} \tau_{a}} F_{l m}-\frac{3 c}{2 R} n_{\gamma l m}(t) .
$$

Since no spontaneous decay of axions feed into these components, they are negligible, as in the previous example. The nonzero axion number density components are given by

$$
\begin{aligned}
& \frac{d n_{a 00}(t)}{d t}+\frac{n_{a 00}}{\tau_{a}}=-\frac{8 \pi^{2}}{\beta m_{a}^{3} \tau_{a}} E_{00}+\frac{8 \pi^{2} \beta}{3 m_{a}^{3} \tau_{a}} F_{00}, \\
& \frac{d n_{a 20}(t)}{d t}+\frac{n_{a 20}}{\tau_{a}}=-\frac{8 \pi^{2}}{\beta m_{a}^{3} \tau_{a}} E_{20}+\frac{8 \pi^{2} \beta}{3 m_{a}^{3} \tau_{a}} F_{20} .
\end{aligned}
$$

Because of (27), this leads to the relation

$$
-\frac{8 \pi^{2}}{\beta m_{a}^{3} \tau_{a}} E_{20}+\frac{8 \pi^{2} \beta}{3 m_{a}^{3} \tau_{a}} F_{20}=\frac{1}{\sqrt{5}}\left(\frac{8 \pi^{2}}{\beta m_{a}^{3} \tau_{a}} E_{00}-\frac{8 \pi^{2} \beta}{3 m_{a}^{3} \tau_{a}} F_{00}\right) .
$$

The photon number density components $n_{\gamma 00}$ and $n_{\gamma 20}$ grow as

$$
\begin{aligned}
\frac{d n_{\gamma 00}(t)}{d t}= & 2 \frac{n_{a 00}}{\tau_{a}}+\frac{16 \pi^{2}}{\beta m_{a}^{3} \tau_{a}} E_{00}-\frac{16 \pi^{2}}{3 m_{a}^{3} \tau_{a}}\left(\beta+\frac{3}{2}\right) F_{00} \\
& -\frac{3 c}{2 R} n_{\gamma 00}(t),
\end{aligned}
$$

and

$$
\begin{aligned}
\frac{d n_{\gamma 20}(t)}{d t}= & 2 \frac{n_{a 20}}{\tau_{a}}+\frac{16 \pi^{2}}{\beta m_{a}^{3} \tau_{a}} E_{20}-\frac{16 \pi^{2}}{3 m_{a}^{3} \tau_{a}}\left(\beta+\frac{3}{2}\right) F_{20} \\
& -\frac{3 c}{2 R} n_{\gamma 20}(t) .
\end{aligned}
$$

Because of (27) and (28), we can combine the previous two equations and write

$$
\begin{aligned}
& \frac{d n_{\gamma 00}(t)}{d t}+\frac{3 c}{2 R} n_{\gamma 00}(t) \\
& =2 \frac{n_{a 00}}{\tau_{a}}+\frac{16 \pi^{2}}{\beta m_{a}^{3} \tau_{a}} E_{00}-\frac{16 \pi^{2}}{3 m_{a}^{3} \tau_{a}}\left(\beta+\frac{3}{2}\right) F_{00}, \\
& \frac{d n_{\gamma 20}(t)}{d t}+\frac{3 c}{2 R} n_{\gamma 20}(t) \\
& =2 \frac{n_{a 00}}{\tau_{a}}\left(\frac{-1}{\sqrt{5}}\right)+\left(\frac{16 \pi^{2}}{\beta m_{a}^{3} \tau_{a}} E_{00}-\frac{16 \pi^{2} \beta}{3 m_{a}^{3} \tau_{a}} F_{00}\right)\left(\frac{-1}{\sqrt{5}}\right), \\
& \quad-\frac{8 \pi^{2}}{m_{a}^{3} \tau_{a}} F_{20} \\
& =\frac{-1}{\sqrt{5}}\left[\frac{d n_{\gamma 00}(t)}{d t}+\frac{3 c}{2 R} n_{\gamma 00}(t)\right]-\frac{8 \pi^{2}}{m_{a}^{3} \tau_{a}} F_{20} .
\end{aligned}
$$

We observe that if the part of backreaction that results in sterile axions is neglected, then

$$
n_{\gamma 20}(t)=-\frac{n_{\gamma 00}(t)}{\sqrt{5}}
$$

so the photons would remain in an $\sin ^{2} \theta$ distribution.

In the previous section, we found that $X \neq Y$; i.e., the angular dependences of axion and photon are not equal. $X=Y$ would have contradicted the results from the previous section, but that is not what this example indicates. This example shows that the combination of 00 and 20 components of axion and photon have the same angular dependence. This is not the same as $X=Y$. There are other photon components besides 00 and 20. These components are neglected under the circumstances that there is no source feeding these components, but they are not equal to 0 . In fact, the presence of these negligible components verifies that the angular dependences cannot be written in a closed form such as $X(\theta)$ or $Y(\theta)$. $X$ is approximately $Y$ but not exactly.

Here we find a similar result to that in [6], which is not a coincidence. We are trying to solve the same type of problems, i.e., we are finding photon distributions from corresponding axion distributions. If the axion distribution is given, then we always have equations for axion components that are absent in the axion distribution. Equations (19) still applies even if the specific axion component is 0 . The solution of these equations with absent axion component is the result of the backreaction of photons somewhat canceling the effect of stimulated decay 
of axion, which prompts simplified equations for the corresponding photon components.

\section{General distribution}

Suppose that we have an axion number density

$$
n_{a}=\Theta(R-r) \sum n_{a l m} Y_{l m}(\Omega)
$$

For any $n_{\text {alm }}=0$, then according to (19) this leads to

$$
F_{l m}=\frac{3}{\beta^{2}} E_{l m} \quad\left(n_{a l m}=0\right) .
$$

Substituting this condition into Eq. (16), we have

$$
\frac{d n_{\gamma l m}(t)}{d t}=-\frac{16 \pi^{2}}{3 m_{a}^{3} \tau_{a}}\left(\frac{3}{2}\right) F_{l m}-\frac{3 c}{2 R} n_{\gamma l m}(t),
$$

and likewise for $n_{\text {alm }}=0$. Hence there is no source feeding those photon components.

The parts of backreaction that results in sterile axions and surface loss are the only terms that contribute to these components. It is expected that these components die out quickly and thus have no effect on lasing. So

$$
n_{\gamma}=\Theta(R-r) \sum n_{\gamma l m} Y_{l m}(\Omega)
$$

where

$$
n_{\gamma l m} \approx 0 \quad\left(\text { when } n_{a l m}=0\right)
$$

In other words, the photon field has the same spherical harmonic components as the axion field, as other components die out quickly due to lack of sources. Neither spontaneous decay nor stimulated decay contributes to the harmonic components of photons that are not present in the axions.

Suppose that all the axion components are nonzero, and they are proportional to each other,

$$
n_{a l m}=\alpha_{l m} n_{a l_{0} m_{0}},
$$

where $\alpha_{l m}$ are numbers and $n_{a l_{0} m_{0}}$ is the fiducial component to which all other components are proportional. Then

$$
\frac{8 \pi^{2} \beta}{3 m_{a}^{3} \tau_{a}} F_{l m}-\frac{8 \pi^{2}}{\beta m_{a}^{3} \tau_{a}} E_{l m}=\frac{d n_{a l m}(t)}{d t}+\frac{n_{a l m}}{\tau_{a}}
$$

and

$$
\begin{aligned}
& \frac{d n_{\gamma l m}(t)}{d t}+\frac{3 c}{2 R} n_{\gamma l m}(t) \\
& \quad=2 \frac{n_{a l m}}{\tau_{a}}+\frac{16 \pi^{2}}{\beta m_{a}^{3} \tau_{a}} E_{l m}-\frac{16 \pi^{2}}{3 m_{a}^{3} \tau_{a}}\left(\beta+\frac{3}{2}\right) F_{l m}, \\
& \quad=-2 \frac{d n_{a l m}(t)}{d t}-\frac{8 \pi^{2}}{m_{a}^{3} \tau_{a}} F_{l m} .
\end{aligned}
$$

If the part of the backreaction that results in sterile axions is neglected, then

$$
\begin{aligned}
\frac{d n_{\gamma l m}(t)}{d t}+\frac{3 c}{2 R} n_{\gamma l m}(t) & =-2 \frac{d n_{a l m}(t)}{d t}=-2 \alpha_{l m} \frac{d n_{a l_{0} m_{0}}(t)}{d t}, \\
& =\alpha_{l m}\left[\frac{d n_{\gamma l_{0} m_{0}}(t)}{d t}+\frac{3 c}{2 R} n_{\gamma l_{0} m_{0}}(t)\right], \\
n_{\gamma l m} & =\alpha_{l m} n_{\gamma l_{0} m_{0}} .
\end{aligned}
$$

Hence the distribution of photons would keep the same shape as that of the axions if sterile axions were neglected.

\section{DISCUSSION}

In the $\sin ^{2} \theta$ distribution example, the 00 and 20 components of the given axion number density are "filled" $(\neq 0)$ but all the other components are "empty" $(=0)$. Our results show that the "filled" photon number density components are also 00 and 20, and the other components are almost "empty" (under circumstances described above). This relationship is not just pertinent to this $\sin ^{2} \theta$ example. In the general distribution, if axions number density components are only "filled" for certain component $l_{0} m_{0}$ with the other components "empty," then the photon number density also has these almost "empty" components, with only $l_{0} m_{0}$ components "filled." This correlation between components of photon and axions also occurs in [6] where the momentum dependences of occupation numbers were considered.

The question of the condition for the lasing to occur has been considered in $[3,7,8,10,20]$. In our formalism, the general conditions under which axion cluster may lase are that the number density of axion needs to be high and that velocity of axion needs to be low. High axion density is required so that stimulated decay can offset the loss of photon due to backreaction and surface loss. Low velocity means $\beta$ is small, which boosts the effect of stimulated decay according to the rate equation. We expect these two conditions are still needed in the analysis here since the characteristics of the seemingly decoupled equations for components have not changed.

The calculation presented here tells one the initial spatial distribution of photons once the spatial distribution of the axions is given. It does not give direct instructions on how to achieve observable effects from axion cluster lasing. The model does display the mechanism that the stimulated 
decay of axion produces the type of photons that have the same momenta as the photons which induced the stimulated decay process. However, there is a compromise made here by using the equation

$$
\begin{aligned}
2 k \frac{d f_{\lambda}(\vec{k})}{d t}= & \frac{4 m_{a} \Gamma_{a}}{\pi} \int \frac{d^{3} k_{1}}{2 k_{1}^{0}} \frac{d^{3} p}{2 p^{0}} \delta^{4}\left(p-k-k_{1}\right) \\
& \times\left\{f_{a}(\vec{p})\left[1+f_{\lambda}(\vec{k})+f_{\lambda}\left(\vec{k}_{1}\right)\right]-f_{\lambda}(\vec{k}) f_{\lambda}\left(\vec{k}_{1}\right)\right\} .
\end{aligned}
$$

The entire model is a local theory. The photon occupation number here and now depends only on particle occupation numbers here and now. If the cluster in the model is a ball and all the quantities are spherical symmetric, the local theory provides useful predictions about the lasing process. However, if the cluster is of some other specific geometrical shape, then the local theory probably will not give pertinent information that reflect the geometry of the cluster. Thus we suggest that a nonlocal lasing theory that could be regulated by some factor $e^{-\Gamma_{a}\left(t-t^{\prime}\right)}$, which takes into account the probability that photons propagating from $\vec{x}^{\prime}$ to $\vec{x}$ without stimulating axion or going to annihilation. In that case the photon occupation number here and now depends on all the past occupation number of events that are casually connected to the photon occupation number here and now.

\section{APPENDIX: PHASE SPACE INTEGRATION OF EVOLUTION EQUATION}

Starting from the evolution relation between axion and photon occupation numbers [3]

$$
\begin{aligned}
\frac{d f_{\lambda}(k)}{d t}= & \frac{m_{a} \Gamma_{a}}{k^{2}} \int_{\frac{m_{a}^{2}}{4 k}} d k_{1} \times\left\{f_{a}\left(k+k_{1}\right)\left[1+f_{\lambda}(k)+f_{\lambda}\left(k_{1}\right)\right]\right. \\
& \left.-f_{\lambda}(k) f_{\lambda}\left(k_{1}\right)\right\},
\end{aligned}
$$

where $f_{\lambda}(k)$ and $f_{\lambda}\left(k_{1}\right)$ are photon occupation numbers of momentum $k$ and $k_{1}$, respectively. Other variables in $f_{\lambda}(k)$ and $f_{\lambda}\left(k_{1}\right)$; i.e., $r, \theta, t$, are the same since they share the same spacetime. $f_{a}\left(k+k_{1}\right)$ is the axion occupation number of momentum $k+k_{1} . \Gamma_{a}$ is the spontaneous axion decay rate. Substitute (1) and (4) into (A1) we arrive at

$$
\begin{aligned}
\frac{d f_{\lambda}(k)}{d t}= & \frac{m_{a} \Gamma_{a}}{k^{2}}\left\{\left[1+f_{\lambda}(k)\right] f_{a c} \Theta(R-r) X(\theta) \times \int_{\frac{m_{a}^{2}}{4 k}} \Theta\left(p_{\max }-\sqrt{\left(k+k_{1}\right)^{2}-m_{a}^{2}}\right) d k_{1}+f_{a c} f_{\lambda c}[\Theta(R-r)]^{2} X(\theta) Y(\theta)\right. \\
& \times \int_{\frac{m_{a}^{2}}{4 k}} \Theta\left(p_{\max }-\sqrt{\left(k+k_{1}\right)^{2}-m_{a}^{2}}\right) \Theta\left(k_{+}-k_{1}\right) \Theta\left(k_{1}-k_{-}\right) d k_{1} \\
& \left.-f_{\lambda}(k) f_{\lambda c} \Theta(R-r) Y(\theta) \int_{\frac{m_{a}^{2}}{4 k}} \Theta\left(k_{+}-k_{1}\right) \Theta\left(k_{1}-k_{-}\right) d k_{1}\right\} .
\end{aligned}
$$

The first and second integrals are the same,

$$
\begin{aligned}
\int_{\frac{m_{a}^{2}}{4 k}} \Theta\left(p_{\max }-\sqrt{\left(k+k_{1}\right)^{2}-m_{a}^{2}}\right) d k_{1} & =\int_{\frac{m_{a}^{2}}{4 k}} \Theta\left(p_{\max }-\sqrt{\left(k+k_{1}\right)^{2}-m_{a}^{2}}\right) \Theta\left(k_{+}-k_{1}\right) \Theta\left(k_{1}-k_{-}\right) d k_{1} \\
& =m_{a} \gamma-k-\frac{m_{a}^{2}}{4 k} .
\end{aligned}
$$

The third integral is related to the backreaction of photons. It is convenient to split it into two parts

$$
\begin{aligned}
\int_{\frac{m_{a}^{2}}{4 k}} \Theta\left(k_{+}-k_{1}\right) \Theta\left(k_{1}-k_{-}\right) d k_{1} & =\int_{\frac{m_{a}^{2}}{4 k}}^{m_{a} \gamma-k} d k_{1}+\int_{m_{a} \gamma-k}^{k_{+}} d k_{1}, \\
& =\left(m_{a} \gamma-k-\frac{m_{a}^{2}}{4 k}\right)+\left(k-k_{-}\right) .
\end{aligned}
$$

The first part represents backreaction resulting in axions with energy less than $m_{a} \gamma$, while the second part gives the backreaction resulting in sterile axions; i.e., where the total energy of the axion $k+k_{1}$ is larger than $m_{a} \gamma$. 
Moving the step function $\Theta(R-r)$ in front of the curly brackets and substituting the results of the integrations, we have

$$
\begin{aligned}
\frac{d f_{\lambda}(k)}{d t}= & \Theta(R-r) \frac{m_{a} \Gamma_{a}}{k^{2}}\left\{\left[1+f_{\lambda}(k)\right] f_{a c} X(\theta)\left(m_{a} \gamma-k-\frac{m_{a}^{2}}{4 k}\right)+f_{a c} f_{\lambda c} X(\theta) Y(\theta)\left(m_{a} \gamma-k-\frac{m_{a}^{2}}{4 k}\right)\right. \\
& \left.-f_{\lambda}(k) f_{\lambda c} Y(\theta)\left[\left(m_{a} \gamma-k-\frac{m_{a}^{2}}{4 k}\right)+\left(k-k_{-}\right)\right]\right\} .
\end{aligned}
$$

This can be written as

$$
\frac{d f_{\lambda}(k)}{d t}=\Theta(R-r) \Theta\left(k_{+}-k\right) \Theta\left(k-k_{-}\right) \frac{m_{a} \Gamma_{a}}{k^{2}}\left\{\left[f_{a c}\left(X+2 f_{\lambda c} X Y\right)-f_{\lambda c}^{2} Y^{2}\right]\left(m_{a} \gamma-k-\frac{m_{a}^{2}}{4 k}\right)-f_{\lambda c}^{2} Y^{2}\left(k-k_{-}\right)\right\} .
$$

The rate of change of photon number density is the integration of this equation over $k$ space

$$
\begin{aligned}
\frac{d n_{\lambda}}{d t} & =\int \frac{d f_{\lambda}(k)}{d t} \frac{d^{3} k}{(2 \pi)^{3}} \\
& =\Theta(R-r) \frac{m_{a} \Gamma_{a}}{2 \pi^{2}}\left\{\left[f_{a c}\left(X+2 f_{\lambda c} X Y\right)-f_{\lambda c}^{2} Y^{2}\right] \times \int_{k_{-}}^{k_{+}}\left(m_{a} \gamma-k-\frac{m_{a}^{2}}{4 k}\right) d k-f_{\lambda c}^{2} Y^{2} \int_{k_{-}}^{k_{+}}\left(k-k_{-}\right) d k\right\} .
\end{aligned}
$$

Evaluating the two integrals,

$$
\begin{aligned}
\int_{k_{-}}^{k_{+}}\left(m_{a} \gamma-k-\frac{m_{a}^{2}}{4 k}\right) d k & =\frac{m_{a}^{2} \gamma^{2} \beta}{2}-\frac{m_{a}^{2}}{4} \ln \left(\frac{1+\beta}{1-\beta}\right), \\
\int_{k_{-}}^{k_{+}}\left(k-k_{-}\right) d k & =\frac{m_{a}^{2} \gamma^{2} \beta^{2}}{2} .
\end{aligned}
$$

gives

$$
\frac{d n_{\lambda}}{d t}=\Theta(R-r) \frac{m_{a}^{3} \Gamma_{a}}{8 \pi^{2}}\left\{\left[f_{a c}\left(X+2 f_{\lambda c} X Y\right)-f_{\lambda c}^{2} Y^{2}\right] \times\left[2 \gamma^{2} \beta-\ln \left(\frac{1+\beta}{1-\beta}\right)\right]-f_{\lambda c}^{2} Y^{2} \times\left(2 \gamma^{2} \beta^{2}\right)\right\}
$$

[1] T. W. Kephart and T. J. Weiler, Phys. Rev. Lett. 58, 171 (1987).

[2] I. I. Tkachev, Phys. Lett. B 191, 41 (1987).

[3] T. W. Kephart and T. J. Weiler, Phys. Rev. D 52, 3226 (1995).

[4] P. Sikivie, Phys. Lett. B 432, 139 (1998).

[5] J. G. Rosa and T. W. Kephart, Phys. Rev. Lett. 120, 231102 (2018).

[6] L. Chen and T. W. Kephart, Phys. Rev. D 101, 103033 (2020).

[7] M. P. Hertzberg and E. D. Schiappacasse, J. Cosmol. Astropart. Phys. 11 (2018) 004.

[8] D. G. Levkov, A. G. Panin, and I. I. Tkachev, Phys. Rev. D 102, 023501 (2020).

[9] T. Ikeda, R. Brito, and V. Cardoso, Phys. Rev. Lett. 122, 081101 (2019).
[10] M. Boskovic, R. Brito, V. Cardoso, T. Ikeda, and H. Witek, Phys. Rev. D 99, 035006 (2019).

[11] J. E. Kim, Phys. Rep. 150, 1 (1987).

[12] H. Y. Cheng, Phys. Rep. 158, 1 (1988).

[13] G. G. Raffelt, Phys. Rep. 198, 1 (1990).

[14] E. W. Kolb and M. S. Turner, The Early Universe (1990), Vol. 69, ISBN 978-0-201-62674-2.

[15] L. D. Duffy and P. Sikivie, Phys. Rev. D 78, 063508 (2008).

[16] E. W. Kolb and I. I. Tkachev, Phys. Rev. Lett. 71, 3051 (1993).

[17] J. V. Sloan et al., Phys. Dark Universe 14, 95 (2016).

[18] E. Braaten and H. Zhang, Rev. Mod. Phys. 91, 041002 (2019).

[19] M. Tanabashi et al. (Particle Data Group), Phys. Rev. D 98, 030001 (2018).

[20] Z. Wang, L. Shao, and L. X. Li, J. Cosmol. Astropart. Phys. 07 (2020) 038. 